\title{
Case based Reasoning for Treatment and Management of Diabetes
}

\author{
Mark K. Kiragu \\ School of Computing and Informatics, \\ University of Nairobi, Kenya
}

\author{
Peter W. Waiganjo \\ School of Computing and Informatics, \\ University of Nairobi , Kenya
}

\begin{abstract}
This research focused on the use of case based reasoning (CBR) for treatment and management of diabetes. CBR is a field of artificial intelligence where one uses past cases as resolution for similar problems. The concept is based on dynamic memory theory where human beings solve problems by recalling encountered cases [1].
\end{abstract}

This research has applied CBR in the field of medicine for treatment and management of diabetes. Diabetes is a family of metabolic disease condition where the patient has elevated blood glucose. There is a rise on the prevalence of diabetes in Kenya with over 2 Million Kenyans suffering from the condition [2]. Damage to nerves, heart failure, kidney failure blindness and amputations are among the diabetes associated complications. Some of key challenges encountered during the management of diabetes include lack of insulin, high cost of drugs, an overworked workforce and low awareness among others.

A formative questionnaire was conducted to find out the viability of previous experience in problem resolution and later a summative questionnaire administered to medical experts to measure the outcome of the research. A prototype was developed using JCOLIBRI framework and trained with a total of 60 cases. 40 cases were type 1 and the remaining 20 cases type 2. A test data of 20 cases was used to measure the accuracy of the system. The key variables used in test were blood glucose, HBA1C (average blood glucose over 3 months), weight and height. The diagnosis predicted by the system was compared against the one obtained by the expert and the results were as follows. When tested with the 3 parameters (Blood Glucose, Height \& Weight) the system had a mean accuracy of $28 \%$ before revision (3rd Cycle of CBR) and after the first revision (3rd Cycle of CBR) the system attained a mean accuracy of $70 \%$ with the 3 parameters. When tested with 1 parameter (Blood Sugar) after revision (3rd Cycle of CBR) the system returned a mean accuracy of $90 \%$ .The accuracy was based on the difference of solution applied between an expert judgment and the system judgment. The level of blood glucose is the key factor to consider during diabetes diagnosis. The research concluded that CBR is more accurate after the revision cycle and as the number of cases increase.

\section{General Terms}

Artificial Intelligent, Formative Evaluation, Summative Evaluation, Database, Drugs, Common Symptoms, Associated Complications, Infections, Investigations, Pathophysiology and Biodata.

\section{Keywords}

Case Based Reasoning, jCOLIBRI Framework,Diabetes,Accuracy,Cases, Retrieve, Reuse ,Revise, Retain, Insulin, Problem, Solution.

\section{INTRODUCTION}

$\mathrm{CBR}$ is a concept that combines problem-solving and integrates learning (from a collection of previous knowledge /experience i.e. cases). CBR has become one of the most successful sub-fields of A.I of recent years [3].It is based on a belief that problems tend to recur. CBR is founded in the works of Roger Schank on dynamic memory [4]. CBR has its background in soft computing methodologies it has been advanced from soft computing methodologies borrowed from artificial Intelligence. A.I is a method of learning gotten from concept representation by use of symbols, learning implementation by use of abstraction, pattern identification use of previous knowledge, natural language processing and data training [5].

Whenever we are presented with a problem we try to recall a previous problem and the steps or methods we used to resolve the problem. We then employ the same set of actions to solve the new problem.

\section{RELATED WORK ON CASE BASED REASONING}

Case based reasoning commonly referred to as CBR, it is a problem solving approach that implements previous experience to solve new problems [6],[7]. CBR has its background in cognitive science, machine learning and knowledge based systems. Presently CBR is established with specific methods and processes together with its application employing those methods for problem resolution in different domains. The concept behind case-base is a repository of previous cases. CBR has been applied in the field of legal reasoning, Planning and decision making in medicine, architecture, performance tuning ,service desk support among others [8],[9],[10],[11],[12],[13],[14],[15],[16],[17].

\subsection{Problem selection in CBR}

To solve a problem similar cases are retrieved and selected and the solutions from the cases are modified or adapted to be used as solution for the new problem [18],[19],[20].

\subsection{Case representation knowledge}

Knowledge is represented as textual, structural and conversational. In structural representation cases are represented to a common structured library. Cases are restricted to represent experience that can be expressed with this vocabulary. In structural representation the features associated to a given case are grouped as flat attribute-value pair in an object-oriented manner as graph structures or set of predicate logic language in a textual CBR cases are represented as free text. This is useful where we have a large collection of case support documentation and the CBR system has a way of searching for appropriate case based on experience. [21]. 


\subsection{Diabetes CBR Application High Level Process Flow}

The process flow is explained in the following steps.

1. Create cases from existing data manual or electronic medical records patient data related to diabetes will be retrieved.

2. New and existing problems will be defined by a set of attributes.

3. The new problem will be matched to an existing cases by use of KNN similarity measurement algorithm

4. Then the case will be scored and assigned a value between 0 and 1 to determine its suitability to deliver care plan.

5. The solution for the matching problem is applied directly or modified to suite the new scenario

6. If no suitable case is found then a new cases is captured hence increasing the competency of the CBR application.

\subsection{CBR Case}

A case comprises of:

a) Problem description: This refers to definition of the problem that requires to be solved. The problem is represented as features or a set of attributes of the case. The features used in this research are common symptoms, physical exam lab results, pathophysiology, biodata, associated complications, and infections.

See Fig. 1 for the problem and solution data model.

b) The Solution is composed of these attributes drugs, insulin, proper diet, exercise, controlled alcohol consumption, social support and type of diabetes.

\subsection{Problem Structuring and analysis mechanism}

Case description can be either described in technical or functional terms. When a complete problem description may not be available this can sometimes lead to ambiguous description which can be eliminated by promoting a dialogue between the user and the retrieval. This method will help the user construct a problem description incrementally through a question answer mechanism [22],[23]

\subsection{Problem Representation and retrieval}

For effective retrieval the users must be in a position to describe the problem that is being resolved.

\subsection{Reasoning in Health Care}

Reasoning undertakes the process of thinking and cognition. It also pertains to thought process, regrouping of ideas and how experience is processed in order to reach a rational conclusion [24].

Steps involved in health reasoning include making expert judgments, the quality of evidence based information to support problem resolution and consideration of whether the levels of evidence available is adequate to commission decisions on diagnostic and treatment options relevant to health care requirements of the patient .

\subsubsection{Reasoning Strategies}

In health thinking there is a relationship between a professional's cognition (thinking), the problem explanation and description and the environment of the situation where cognition is applied. Making judgments on the use of evidence based on past experience but also on hypothetical knowledge, judging patient's situation, hypotheses generation, diagnostic reasoning and reflection forms part of the multiple cognitive processes needed for health care management. Several frameworks have been used to collaborate in decision making and service organization in responses to important patient's needs interpretation signals. The physiological assessment of the patient is dependent on technology and precise information provided by these technologies.

\subsection{Similarity theory in CBR}

Similarity is a function sim:PxP $->[0,1]$ which compares two problem features from $\mathrm{P}$ and returns a similarity assessment as a real value from $[0,1]$ a high value confirms a high similarity. For a new Problem $\mathrm{P}$ a case $\mathrm{cl}=(\mathrm{p} 1, \mathrm{~s} 1)$ is preferred over $\mathrm{c} 2=(\mathrm{p} 2, \mathrm{~s} 2) . \mathrm{c} 1>\mathrm{pc} 2$ iff $\operatorname{sim}(\mathrm{p}, \mathrm{p} 1)>\operatorname{sim}(\mathrm{p}, \mathrm{p} 2)$ the similarity based retrieval lists $\mathrm{c} 1$ before $\mathrm{c} 2$ and if the utility $\mathrm{s} 1$ for solving $\mathrm{p}$ is higher than utility $\mathrm{s} 2$ for solving $\mathrm{p}$ case $\mathrm{c} 1$ should be preferred over. CBR also applies K- Nearest Neighbour retrieval between 2 cases among other similarity notions and functions that converts the summation of inputs and their respective weights to a real value between 0 and 1 [25],[26],[27].

\subsubsection{Highest level of CBR abstraction}

The highest level of abstraction of CBR is described by a cycle which involves four processes [28],[29],[30],[31],[32].

a. Retrieve/selecting the most similar case or cases.

b. Reuse the information and knowledge in that case to solve a problem this involves copying a past solution.

c. Revise the proposed solution this process involves the modification or update of the copied solution to fit the current Problem. The revision part may involve an expert intervention.

d. Retain the experience likely to be useful for future problem solving this may include indexing for quick retrieval where we have a large number of cases is searched.

\subsection{Diabetes}

According to Centres for Disease Control diabetes is a condition where blood glucose is too high above normal levels.

\subsubsection{Prediabetes}

Is due to blood sugar being above normal levels but not high enough to cause diabetes.

\subsubsection{Type 1 diabetes}

This type of diabetes (Juvenile diabetes) develops often in young people however it can also develop in adults. In this case the pancreas does not produce enough or no insulin at all as result of destruction of pancreases beta cells by the body autoimmune system [33],[34],[35].

\subsubsection{Type 2 diabetes}

Type 2 diabetes also called adult onset affects people at any age including children 


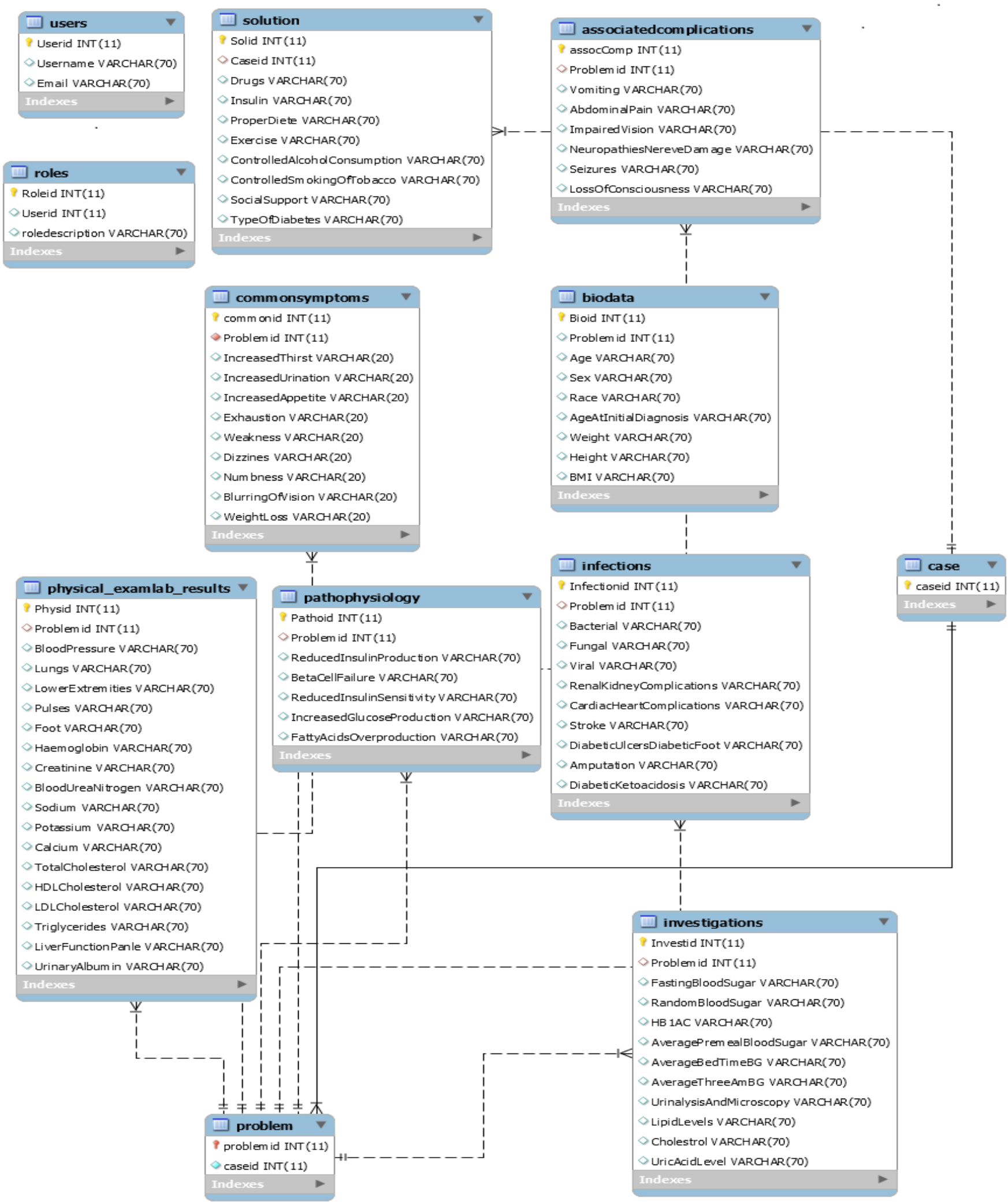

Fig 1: Diabetes CBR Data Model

\subsubsection{Gestational diabetes}

This type of diabetes develops during expectancy.

\subsubsection{Kenyans faces rising burden of diabetes}

WHO shows the cost of healthcare in Kenya is an obstacle and complicates treatment .Diabetes has become a growing problem in developing countries, an increase largely driven by a rising obesity see Fig $2.80 \%$ of an estimate of 1.5 million global diabetes deaths in 2012 occurred in low and middle income countries. According to WHO Health data 2012, $1 \%$ of the Kenyan deaths were attributable to diabetes. Overtime diabetes damages the heart, blood vessels, eyes, kidneys and nerves causing chronic problems and early death [36],[37]. 


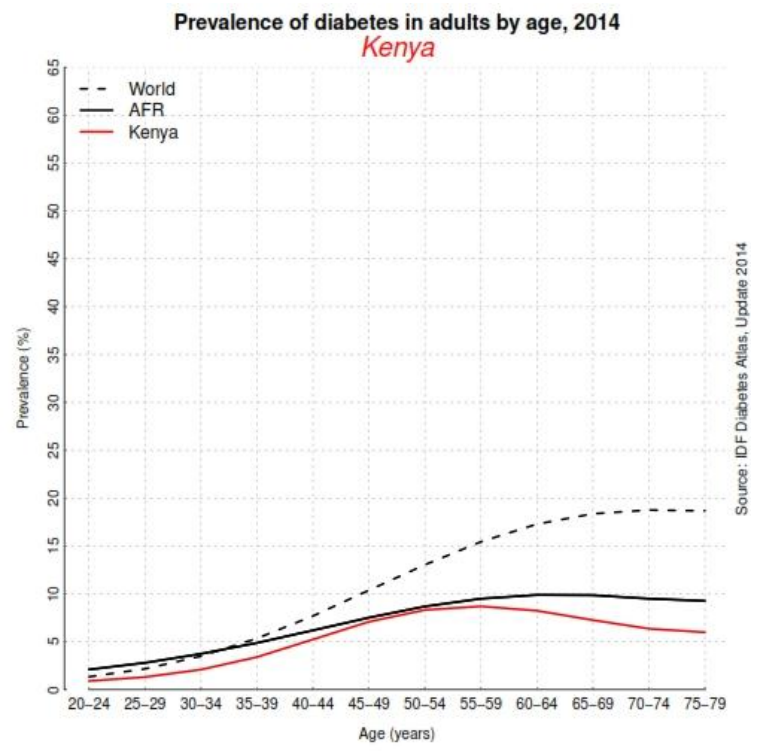

Figure 2: Prevalence of Diabetes in Kenya (source: Internal Diabetes Federation

https://www.idf.org/membership/afr/kenya)

\section{METHODOLOGY}

The method used for this research had 3 main sections. 1) Formative study, 2) Development of the CBR prototype 3) Summative study. 1). The principle steps included:

1) Capturing of previous diabetic cases .

2) Formatting and cleaning of the data to represent diabetes problem features and solution features. The output of this process led to a case which comprised of two parts

i) A diabetic problem description.

ii) A solution description.

3) Design of the system.

4) A case based reasoning application development by use of java workbench JCOLIBRI framework

5) Implementation of the system and deployment.

6) Development of the test cases to demonstrate the following. Retrieval, reuse, revision \& retention.

7) Evaluation of the test cases

8) Summative evaluation to determine the outcome of the results of accuracy on whether a CBR tool can be used to diagnose, treat $\&$ manage diabetes based on the number of cases tested

\subsection{Formative study}

A study was conducted with a sample size of 14 medical personnel. This survey was meant to find out whether the use of previous knowledge and experience can be applied to resolve the new diabetic problems. The research sample comprised of diabetologists, general practitioners, pharmacists and nurses from Kenyatta National Hospital and Nairobi Women's Hospital. The research also included getting feedback from some of the organizations responsible for management, control and awareness of diabetes in Kenya .The organizations are Diabetes Association of Kenya and Diabetes management institute.

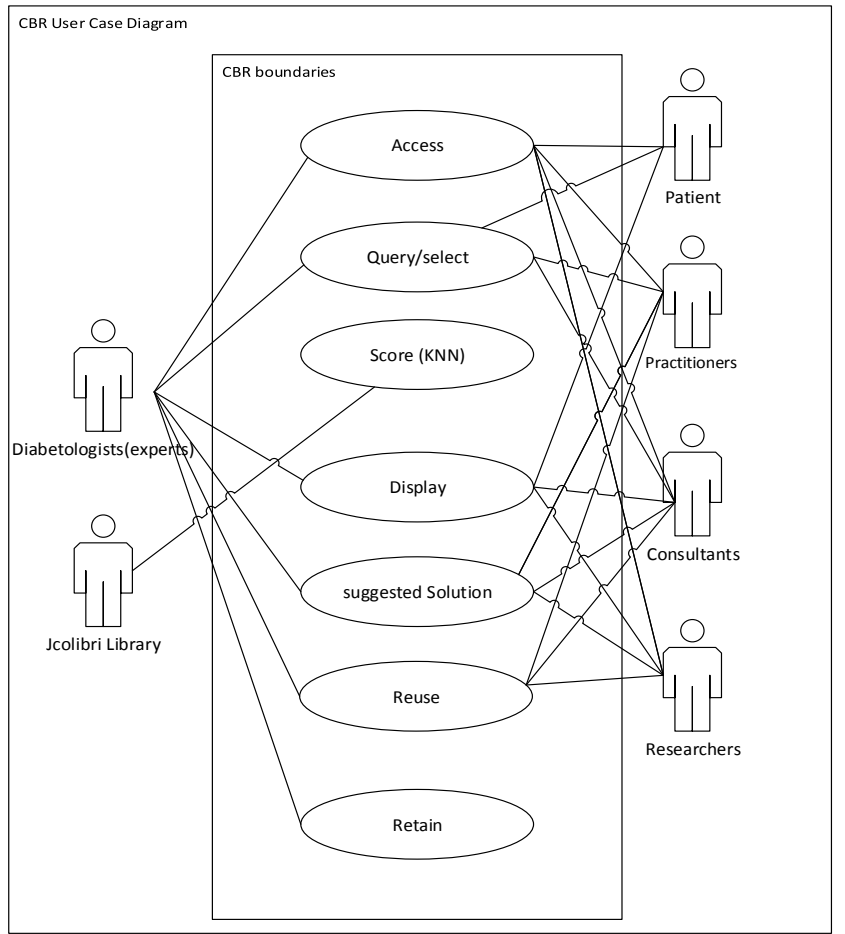

Fig 3 Architectural Design

1) Medical Expert

Is the Diabetes specialist who will be responsible for validating the learned cases the expert will be the super user for the system.

\section{2) User Interface}

The Interface is the JAVA GUI that will provide dialogs to execute the $4 \mathrm{R}$ cycle.

\section{3) Primary Functions}

The Primary Functions includes the selection of the cases from the database based on the nearest neighbor algorithm list the cases and display them in a descending version starting with the most similar to the least similar.

\section{4) Similarity Functions}

The KNN algorithm will be used to compare the previous cases and target cases.

\section{5) Hibernate}

It's a Java framework used to map java objects to database objects by use of xml files.

\subsection{MySQL Database}

The MySQL is an RDBMS database that will be used for persistence storage of the diabetes cases. The case base will be stored in a database. The database used will either be an RDBMS or a text file as JCOLIBRI uses internally the hibernate as the middle ware technology. Hibernate supports high performance objects and relational persistence and query services. It also supports Jboss,J2ee server supporting different databases and xmls files. By implementing hibernate JCOLIBRI will allow the use of RDBMS databases.

\subsection{Diabetes Feature Description and representation}

A set of attributes that describes diabetes will be captured. These attributes will be used to generate the diabetes cases structure. The attributes will be represented by use of data objects which will include both typical data types such as integer, real, Boolean or defined types. This process shall 
involve the representation of cases (diabetic instances) as java objects referred to as java beans with get and set methods. In jCOLIBRI it's possible to create cases as normal java classes. This capability simplifies programming, debugging and configuration of CBR applications by use of frameworks you can generate GUI and automatic persistence's.

\subsection{Summative study}

Summative evaluation was carried out to determine the outcome of the results of accuracy on whether a CBR tool can be used to diagnose, treat \& manage diabetes based on the number of cases tested. The evaluations were conducted by medical experts (diabetologists, general practitioners, pharmacists, clinical officers and nurses). Experts from Diabetes Association of Kenya and Diabetes Management Institute participated in the evaluation feedback.
The experts undertook the following activities: training of the tool with previous cases of diabetes, recording the cases and saving the case in a persistent memory, used the trained system to dragonize and make clinical judgments of new cases based on the similarity of previous cases, tested on ease of use, usefulness of the tool, attitude to use the system, areas to be improved, parts of the software to be added or removed, the user experience of the interface and the functionality of the system.

\section{RESULTS AND DISCUSSION} 4.1 Reasoning Based on Experience.

The use of previous experience can be used to support health decisions in treatment and management of diabetes

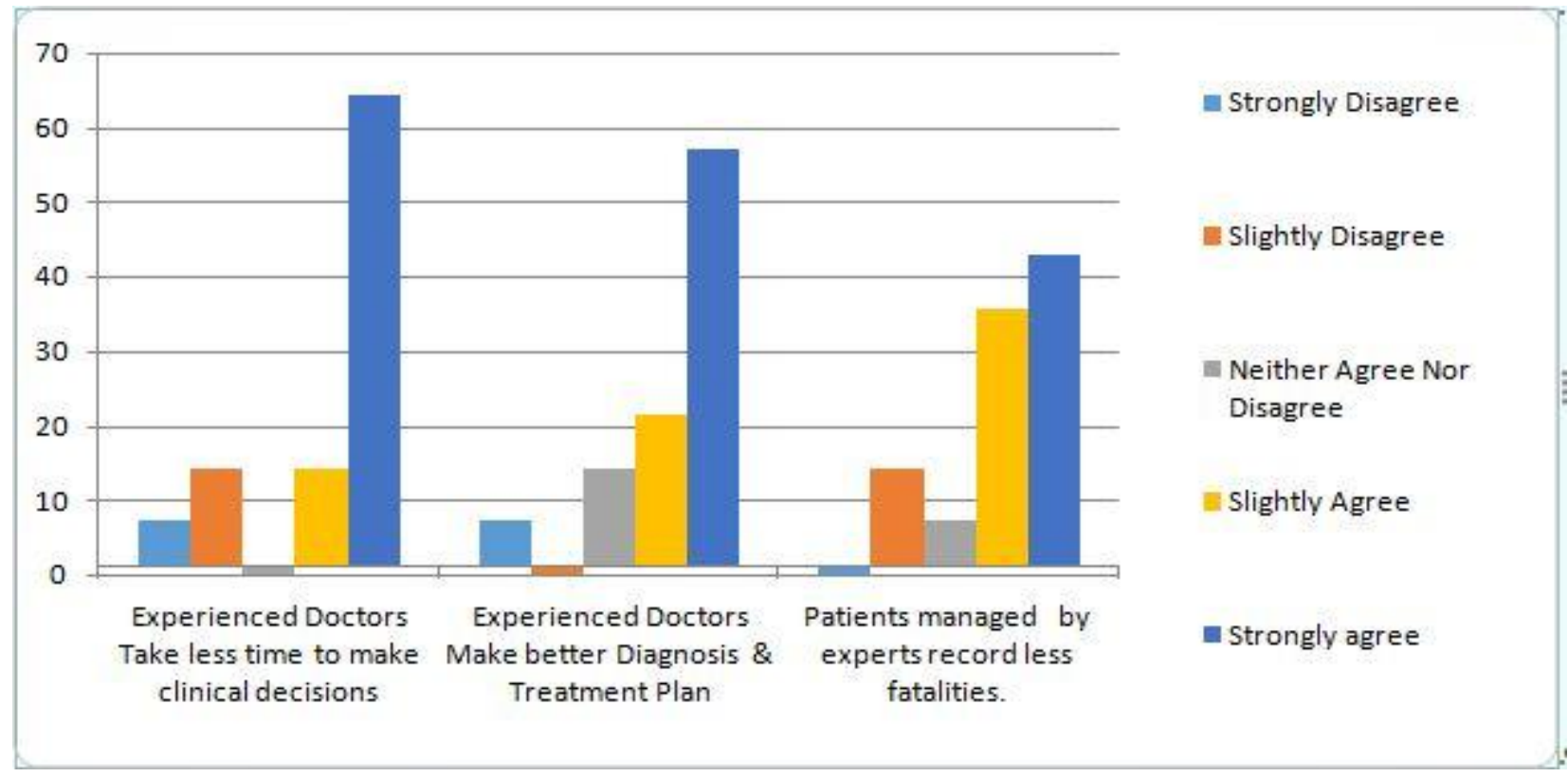

Fig. 4 Use of Experience for making clinical decisions.

The research found out that the previous experience can be used to support health decisions in treatment and management of diabetes $78.58 \%$ (11/14.) agreed that Experienced Doctors take less time to make clinical decisions $78.57 \%(11 / 14)$ agreed that Experienced Doctors Make better Diagnosis \& Treatment Plan and $78.57 \%(11 / 14)$ agreed that Patients managed by expert's record fewer fatalities refer to Fig 4 for chart display.

A sample application's $3^{\text {rd }}$ cycle screen output illustrated in Fig 5.

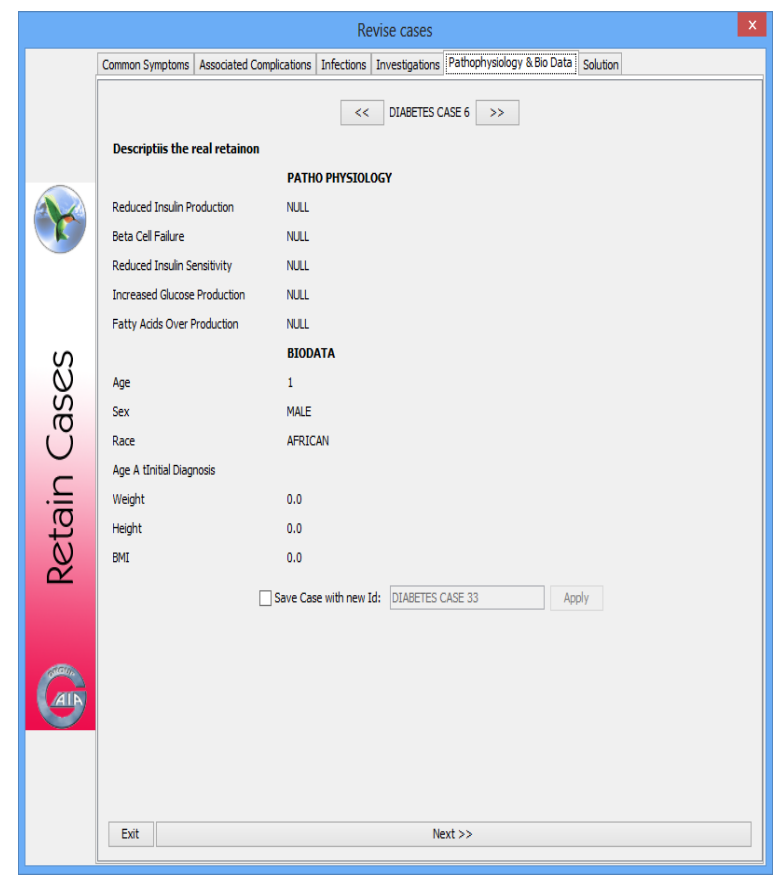

Fig. 5 developed CBR prototype $3^{\text {rd }}$ cycle screen output. 


\subsection{Prototype Results}

A prototype was developed using JCOLIBRI framework and trained with a total of 60 cases. 40 cases were type 1 and the remaining 20 cases type 2 . A test data of 20 cases was used to measure the accuracy of the system. The key variables used in test were blood glucose, HBA1C (average blood glucose over 3 months), weight and height. The diagnosis predicted by the system was compared against the one obtained by the expert and the results were as follows .The system had a mean accuracy of $28 \%$ before revision and after the first revision the system attained a mean accuracy of $70 \%$. The accuracy was based on the difference between an expert judgment and a system judgment. The average similarity before revision for the 3 parameters (blood sugar/average blood sugar, weight and height) is $\mathbf{0 . 2 8}$ the average blood sugar was used where blood sugar value was not captured or unavailable. The average similarity after revision for the 3 parameters (blood sugar/average blood sugar, weight and height ) of $\mathbf{0 . 7 0}$ was less than the similarity for 1 parameter (blood sugar/average blood sugar) 0.9 proving that Blood sugar or average blood sugar is the key factor for diabetes diagnosis. The average blood sugar was used where blood sugar value was not captured or unavailable.

Table 1 Training Data Used.( The Data is real cases captured from previous experience) some data was not available hence the blanks

\begin{tabular}{|c|c|c|c|c|c|c|c|}
\hline Blood Sugar & Average & Weight & Height & HBA1C(average blood sugar over 3 months) & Served By & Sign & Insulin Dosage \\
\hline 0 & 12.8 & - & - & - & - & - & $12 / 8$ \\
\hline 10.9 & - & - & - & 10.5 & - & - & $12 / 8$ \\
\hline 5.3 & 11.7 & - & - & - & - & - & $12 / 8$ \\
\hline 8.8 & 11.2 & 25 & 136 & 14.1 & - & - & $12 / 8$ \\
\hline - & - & - & 144 & 11.1 & - & - & $18 / 12$ \\
\hline 11.5 & - & 28 & 145 & 12.4 & - & - & $16 / 10$ \\
\hline 15 & - & 53 & 159 & & - & - & $32 / 18$ \\
\hline 18.1 & - & 58 & 159 & & - & 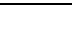 & $35 / 18$ \\
\hline 15.8 & - & 58 & 159 & & - & - & $35 / 25$ \\
\hline 13.3 & - & 61 & 159 & & - & - & $35 / 25$ \\
\hline 19.1 & - & - & - & & - & - & $30 / 25$ \\
\hline 14 & 22.9 & - & - & & - & - & $30 / 25$ \\
\hline- & - & - & - & & - & - & $30 / 25$ \\
\hline- & - & - & - & & - & - & $25 / 20$ \\
\hline - & - & - & - & & & & $35 / 30$ \\
\hline 16.6 & - & 71 & 164 & $>13$ & & & $22 / 25$ \\
\hline 15.6 & - & 35 & 152 & 10.4 & & & $16 / 14$ \\
\hline 0 & - & - & - & & & & $16 / 14$ \\
\hline 8.7 & - & 37 & 152 & 11.9 & & & $22 / 20$ \\
\hline - & 11.1 & - & - & & & & $22 / 20$ \\
\hline 11.3 & 6.9 & - & - & & & & $22 / 20$ \\
\hline 14.9 & 9.6 & 41 & 157 & 13.6 & & & $30 / 16$ \\
\hline - & - & - & - & & & & $32 / 16$ \\
\hline- & 0 & 0 & 0 & & & & $32 / 16$ \\
\hline 12 & 0 & 43 & 158 & 12.8 & & & $32 / 16$ \\
\hline 0 & 0 & 0 & 0 & & & & $35 / 25$ \\
\hline 0 & 0 & 0 & 0 & & & & $22 / 20$ \\
\hline 16.5 & 6.8 & 44 & 159 & & & & $22 / 20$ \\
\hline 0 & 0 & 0 & 0 & & & & $28 / 14$ \\
\hline 23.5 & 8.6 & 51 & 162 & 9.2 & & & $28 / 22$ \\
\hline 16.1 & 0 & 52 & 161 & 9.1 & & & $30 / 20$ \\
\hline 0 & 0 & 0 & 0 & & & & $30 / 20$ \\
\hline 0 & 0 & 0 & 0 & & & & $30 / 20$ \\
\hline 18.9 & 18.1 & 35 & 145 & & & & $30 / 20$ \\
\hline 18.1 & 16.1 & 37 & 145 & 9.5 & & & $30 / 20$ \\
\hline
\end{tabular}




\begin{tabular}{|c|c|c|c|c|c|}
\hline 2.8 & 5.2 & 40 & 148 & 12.2 & $34 / 20$ \\
\hline 11.9 & 18.7 & 44 & 152 & & $32 / 18$ \\
\hline 0 & & & & & $32 / 18$ \\
\hline 18.8 & & 47 & 154 & 9.8 & $30 / 18$ \\
\hline 0 & & & & & $31 / 20$ \\
\hline 7.4 & & & & & $31 / 20$ \\
\hline 0 & & & & & $32 / 18$ \\
\hline 3.6 & & 54 & 157 & 11.3 & $32 / 18$ \\
\hline 10.9 & & 56 & 159 & 12.5 & $32 / 18$ \\
\hline 5.1 & & 78 & 180 & 13.7 & $36 / 26$ \\
\hline 5.2 & & 78 & 181 & 14 & $36 / 28$ \\
\hline 8.5 & & 77 & 181 & 7.14 & $36 / 20$ \\
\hline 14.1 & & 76 & 181 & & $36 / 20$ \\
\hline 10 & & 73 & 181 & & $20 / 18$ \\
\hline 7.2 & & & & & $26 / 13$ \\
\hline 7.1 & & & & & $26 / 13$ \\
\hline 3.6 & & 75 & 181 & 12.6 & $28 / 18$ \\
\hline 4.6 & & 79 & 181 & 9.2 & $35 / 18$ \\
\hline 26.8 & $40 / 20$ & 44 & 155 & & \\
\hline
\end{tabular}

Table 2 Average Percentage Accuracy before the Revision $\left(3^{\text {rd }}\right)$ Cycle is $(28 \%)$ for 3 parameters (weight, height and blood sugar/average blood sugar).

The average similarity before revision for the 3 parameters (blood sugar/average blood sugar, weight and height) is $\mathbf{0 . 2 8}$ the average blood sugar was used where blood sugar value was not captured or unavailable.

\begin{tabular}{|c|c|c|c|c|c|c|c|c|c|c|}
\hline $\begin{array}{l}\text { Blood } \\
\text { Sugar }\end{array}$ & $\begin{array}{l}\text { Average } \\
\text { Blood } \\
\text { Sugar }\end{array}$ & Weight & Height & HBA1C & $\begin{array}{l}\text { Expert } \\
\text { Insulin } \\
\text { DOSAGE }\end{array}$ & $\begin{array}{l}\text { System } \\
\text { Insulin } \\
\text { Dosage } \\
\text { with all } 3 \\
\text { parameters }\end{array}$ & $\begin{array}{l}\text { similarity } \\
\text { with } 3 \\
\text { params }\end{array}$ & $\begin{array}{l}\text { Accuracy } \\
\text { with } 3 \\
\text { params in } \\
\%\end{array}$ & $\begin{array}{l}\text { Accuracy } \\
\text { with } \\
\text { height } \\
\text { only }\end{array}$ & $\begin{array}{l}\text { Accuracy } \\
\text { Weight } \\
\text { Only }\end{array}$ \\
\hline 12.8 & 12.8 & & & & $12 / 8$ & $32 / 18$ & 0.7 & $30 \%$ & & \\
\hline 7.6 & & 26 & 145 & 10.3 & $18 / 12$ & $34 / 20$ & 0.8 & $20 \%$ & $16 / 10(1.0) 0 \%$ & No Sol.(0.0) $0 \%$ \\
\hline 8.0 & & 56 & 159 & & $35 / 15$ & $34 / 20$ & 0.8 & $20 \%$ & $32 / 18(1.0) 0 \%$ & $32 / 18(1.0) 0 \%$ \\
\hline & 15.2 & & & & $25 / 16$ & $32 / 18$ & 0.7 & $30 \%$ & & \\
\hline 12.1 & 17.3 & 63 & & 7.14 & $30 / 25$ & $26 / 13$ & 0.646 & $40 \%$ & & \\
\hline 24.6 & & 70 & 163 & $>13$ & $30 / 25$ & $34 / 20$ & 0.8 & $20 \%$ & No Sol. $0(0 \%)$ & No Sol. (0.0) 0\% \\
\hline 14.3 & 7.1 & 41 & 159 & 9.9 & $35 / 25$ & $35 / 18$ & 0.6 & $40 \%$ & $32 / 18(1.0) 0 \%$ & $30 / 16(1.0) 0 \%$ \\
\hline 4.5 & 18.1 & 35 & 145 & 12.1 & $30 / 20$ & $34 / 20$ & 0.8 & $20 \%$ & $16 / 10(1.0) 0 \%$ & $16 / 14(1.0) 0 \%$ \\
\hline & & & & & $31 / 20$ & $28 / 18$ & 0.7 & $30 \%$ & & \\
\hline 7.0 & & 79 & 180 & 11.7 & $38 / 26$ & & & $30 \%$ & No Sol (0.0) 0\% & $35 / 18(1.0) 0 \%$ \\
\hline 15.9 & $40 / 20$ & 47 & 153 & & & & & $28 \%$ & No Sol $(0.0) 0 \%$ & $30 / 18(1.0)$ \\
\hline
\end{tabular}


Table 3 Average Percentage Accuracy after the Revision $\left(3^{\text {rd }}\right)$ Cycle is (70\%) for 3 parameters (weight, height and blood sugar/average blood sugar) and $90 \%$ for 1 parameter (blood sugar/ average blood sugar).

The average similarity after revision for the 3 parameters (blood sugar/average blood sugar, weight and height ) of $\mathbf{0 . 7 0}$ was less than the similarity for 1 parameter (blood sugar/average blood sugar) 0.9 proving that Blood sugar or average blood sugar is the key factor for diabetes diagnosis. The average blood sugar was used where blood sugar value was not captured or unavailable.

The Insulin dosage suggested for the 10 cases by the system was the same as the expert dosage after $3^{\text {rd }}$ cycle of revision. The KNN similarity method was used.

\begin{tabular}{|c|c|c|c|c|c|c|c|c|c|c|c|}
\hline 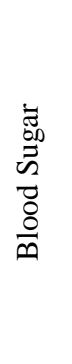 & 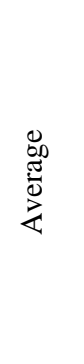 & $\begin{array}{l}\frac{\vec{D}}{50} \\
\frac{\overrightarrow{0}}{3}\end{array}$ & $\begin{array}{l}\frac{\vec{D}}{000} \\
\frac{0}{\mathbb{I}}\end{array}$ & $\begin{array}{l}\underset{\mathbb{Z}}{U} \\
\text { 壬 }\end{array}$ & 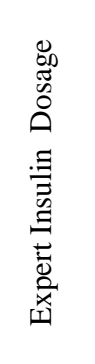 & 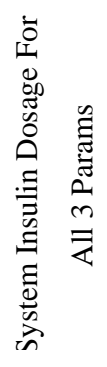 & 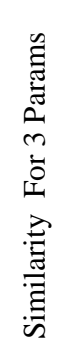 & 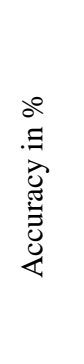 & 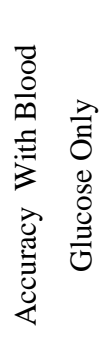 & 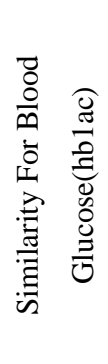 & 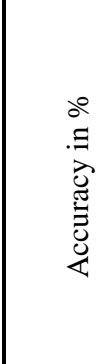 \\
\hline 8.8 & 11.2 & 25 & 136 & 14.1 & $12 / 8$ & $12 / 8$ & 0.75 & $75 \%$ & $12 / 8$ & 1.0 & $100 \%$ \\
\hline 11.5 & 11.5 & 28 & 145 & 12.4 & $16 / 10$ & $16 / 10$ & 0.75 & $75 \%$ & $16 / 10$ & 1.0 & $100 \%$ \\
\hline 15 & 15 & 53 & 159 & 15 & $32 / 18$ & $32 / 18$ & 0.75 & $75 \%$ & $32 / 18$ & 1.0 & $100 \%$ \\
\hline 18.1 & 18.1 & 58 & 159 & 18.1 & $35 / 18$ & $35 / 18$ & 0.5 & $50 \%$ & $35 / 18$ & 1.0 & $100 \%$ \\
\hline 18.1 & 15.8 & 58 & 159 & 15.8 & $35 / 25$ & $35 / 18$ & 0.75 & $75 \%$ & $35 / 25$ & 1.0 & $100 \%$ \\
\hline 13.3 & 13.3 & 61 & 159 & 13.3 & $35 / 25$ & $35 / 25$ & 0.75 & $75 \%$ & $35 / 25$ & 1.0 & $100 \%$ \\
\hline 19.1 & 19.1 & 0 & 0 & 19.1 & $30 / 25$ & $30 / 25$ & 0.75 & $75 \%$ & $30 / 25$ & 1.0 & $100 \%$ \\
\hline 14 & 22.9 & 0 & 0 & 18.4 & $30 / 25$ & $30 / 25$ & 0.75 & $75 \%$ & No sol. & 0.0 & $0 \%$ \\
\hline 16.6 & 16.6 & 71 & 164 & 16.6 & $22 / 25$ & $22 / 25$ & 0.75 & $75 \%$ & $22 / 25$ & 1.0 & $100 \%$ \\
\hline 15.6 & 15.6 & 35 & 152 & 10.4 & $16 / 14$ & $16 / 14$ & 0.5 & $50 \%$ & $16 / 14$ & 1.0 & $100 \%$ \\
\hline & & & & & & & & $70 \%$ & & & $90 \%$ \\
\hline
\end{tabular}

\subsection{Summative evaluation}

$60 \%$ of the experts agreed that the software was easy to use and $70 \%$ agreed that it was interesting to use. 


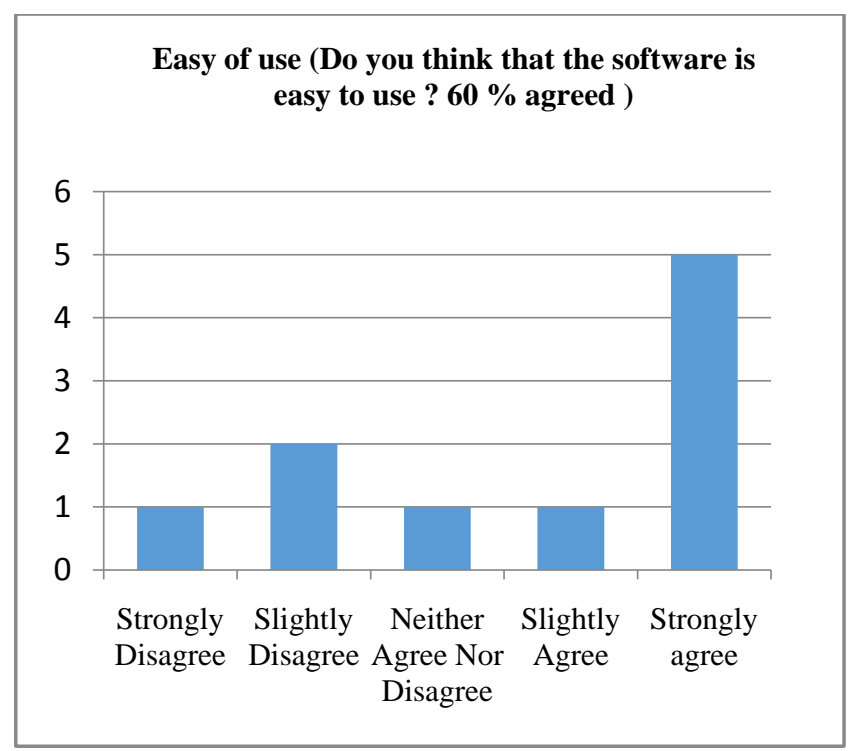

\subsection{Features to be added}

1) The users recommended a mobile version and a web based version to enhance accessibility to the system.

2) Management of complications of the diabetes and diabetic related cases

3) Integration to the existing hospital system to book patients after seeing them.

\subsection{Most Useful Features of the System}

From the participant's response case resolution and management, signs and symptoms were the most useful features of the system.

\section{CONCLUSION AND RECOMMENDATION}

A CBR Application was developed for treatment and management of diabetes using jCOLBIRI CBR framework. The application was meant to demonstrate reasoning by use of previously experienced past events. The system employed case based methodology of reasoning which involves the $4 \mathrm{R}$ process. The success of the system relied on use of a similarity matching between the captured and revised cases and the new case. A case in this context implies a problem solution pair. The problem consists of diabetes problem description (symptoms) and its solution. The problem structure was defined by a medical expert. The system was deployed and tested with real life cases and then updated by a medical expert. The CBR concept implemented had an accuracy of $75 \%$ when tested with 3 parameters (Blood glucose, height \& weight) and an accuracy of $90 \%$ when tested with 1 parameter (blood glucose) after the revision cycle ( 3 rd cycle of CBR), thus proving that the competence of the CBR system increases after the $3^{\text {rd }}$ cycle (Revision) and as the number of cases increase .The accuracy of $90 \%$ leads us to conclude that the key factor to consider when treating diabetes is the level of blood glucose. The accuracy of the system can further be improved by combining different pattern matching algorithms such as (Euclidean, Hamming distance, neural networks etc.) and building a bigger case base.

\section{The major observations of the study were that the}

i) CBR accuracy is highest after the revision (3rd cycle).

ii) The blood sugar level is the key determinant factor prove of diabetes.

\section{ACKNOWLEDGMENTS}

Appreciation goes to the school of computing and informatics university of Nairobi and Co-author prof. Wagacha for his guidance during the entire work.

\section{REFERENCES}

[1] Aamodt, A.,Plaza, E. (1994).Case-Based Reasoning:Foundational Issues,Methodological Variations, and System Approaches

[2] Medical Journal of Therapeutics(2008) Obstacle to Diabetes Care in Kenya.

[3] Wei-Fan, C., and Kuo-Chuan, Y.(2006). Creating a Case-Based Reasoning Digital Library to Improve Learning in an Introductory Programming Course.

[4] Craig, S. and David, W.(2003).Toward memory-based reasoning.

[5] Watson,I. (1999). Case-based resoning is a methodology not a technology

[6] Adebayo, K.,Adekoya, A. and Ekwonna, C. (2014).Temperament and Mood Detection Using CaseBased Reasoning.

[7] Antti, V., Olli, A. and Eero, H. Combining Case-Based Reasoning and Semantic Indexing in a Question-Answer Service.

[8] Ashok, K. and Craw2, S. Design, innovation and casebased reasoning.

[9] Breese, J.(1994). Decision-Theoretic Case-Based Reasoning.

[10] Mariana, M. and Ernesto, O.(2010).Integration of Rule Based Expert Systems and Case Based Reasoning in an Acute Bacterial Meningitis Clinical Decision Support System.

[11] Shahina, B. and Peter, F. (2009).Case-based systems in health sciences - a case study in the field of stress management.

[12] Sarah,J. (2006).Using Case-Based Reasoning for Spam Filtering.

[13] Salha, B. Abdullah(1997) The fundamentals of casebased reasoning: application to a building defect problem.

[14] Surjeet, D. and Dr. Vijay.(2011).Case Retrieval Optimization of Case-based reasoning through Knowledge-Intensive Similarity Measures.

[15] Ting-Peng, L. Analogical reasoning and case-based learning in model management systems.

[16] Zouhair, A. ,Bertelle, C. (2012). Dynamic Case-Based Reasoning Based on the Multi-Agent Systems: Individualized Follow-Up of Learners in Distance Learning.

[17] Sima, S. (2013).Case-Based Reasoning for Diagnosis and Solution Planning.

[18] A-Xing, Z., James, E. Burt . A Case-based Reasoning Approach to Fuzzy Soil Mapping. 
[19] Burke1, B. , MacCarthy2, S. and Petrovic1, R.(2001). Case-based Reasoning in Course Timetabling: An Attribute Graph Approach.

[20] Ralph, B. , Klaus-Dieter, A. ,Mirjam, M. , Meike, R. and Kerstin, B.(2009). Case-Based Reasoning. 20

[21] Roger, C. and Alex, K., Christopher, K.(2014). Inside Case-Based Explanation.

[22] Hans-Dieter, B. and Michael, M. On the Notion of Similarity in Case Based Reasoning and Fuzzy Theory.

[23] Watson and Kolodner, J. (2009).An Introduction to CaseBased Reasoning.

[24] Maggin, B.(2007). Clinical reasoning and its application to nursing: Concepts and research studies.

[25] Sima,S.( 2013) Case-Based Reasoning for Diagnosis and Solution PlanningCase-Based Reasoning for Diagnosis and Solution Planning.

[26] Hugh, O. and Derek, B. Models of Similarity for CaseBased Reasoning.

[27] Petri, M. \& Henry, T. Bayesian Case-Based Reasoning with Neural Networks

[28] Henry, P., Adam, W. and Katie, A. (2013). A formalization of argumentation schemes for legal casebased reasoning in ASPIC.Hinkle, D. and Toomey, C. (1995). Applying Case-Based Reasoning to Manufacturing.
[29] Hyowon, S. \& Jae, H.(2008). Ontology-based CaseBased Reasoning (OntCBR) for Engineering Design. Iain, B.(2006). A case-based reasoning method for fixture design.

[30] Jaroslav, H., Jirı, D.(2008).Using case-based reasoning for mobile robot path planning.

[31] Juan A.(2013). jcolibri2: A framework for building Casebased reasoning systems

[32] Roth-Berghofer T.(2012), Building Case-based Reasoning Applications with myCBR and COLIBRI

[33] Rombo,O.(2013). Diabetes And Blindness In Kenya: A chronic diseases of nutrition in transition

[34] Kiberenge, M. (2010).Knowledge , Attitude and Practices Related to Diabetes Among Community Members in Four Provinces in Kenya: a Cross-Sectional Study

[35] Health Improvement Scotland (2013) Management of Diabetes A National Clinical Guideline

[36] World Diabetes Foundation. National Diabetes ProgrammeWDF09-436 .(2015).Available: <http://www.worlddiabetesfoundation.org/projects/kenya -wdf09-436>.

[37] World Health Organization .(2014).Available http://www.who.int/features/2014/kenya-risingdiabwetes/en/. 